\title{
SOME URGENT PROGRAMMES OF MERIDIAN OBSERVATIONS ESPECIALLY FOR THE SOUTHERN HEMISPHERE
}

\author{
(Invited Paper)
}

M. S. ZVEREV

Pulkovo Observatory, Leningrad, U.S.S.R.

\begin{abstract}
Recommendations of Commission 8 at previous IAU General Assemblies on the following programmes are recalled: Bright Stars (BS), Double Stars unsuitable for photographic observations (DS), Latitude Stars (LS) and Zodiacal Stars (ZS). One more programme - 'Additional Reference Stars in the areas with (ialaxies of the Pulkovo Programme' (PS) containing 735 stars for the whole sky is proposed.
\end{abstract}

For the observers with meridian instruments there exist the following main problems:

(1) Observations of fundamental stars - bright (FK4) and faint (including FKSZ) for improving the fundamental system of coordinates and proper motions. Absolute observations of these stars are of special importance.

(2) Regular observations of the Sun and planets (major and some of the minor ones) and also the Moon - for improvement of orbits and orientation elements for the fundamental system. Differential observations in the fundamental catalogue system are preferable.

(3) Differential determinations of coordinates of the reference stars for photographic astrometry. We think that the totality of the AGK3R + SRS stars can be named 'international reference stars' (IRS). This program contains about 41000 stars for the whole sky, i.e. on the average one star per square degree.

Besides the above problems there are some other urgent tasks in Meridian Astronomy. Some of them were discussed at previous IAU Assemblies and the recommendations for observatories to participate in observations were adopted. However these recommendations have been insufficiently realized. I should like to recall some of them and to add one more program.

\section{Bright Stars (BS)}

Resolution No. 17. of the Xth Assembly of the IAU (Moscow, 1958) recommended making meridian observations of all bright stars up to 6.00; the XIth IAU Assembly (Berkeley, 1961) recommended (resolution No. 7 of Commission 8) observing the southern bright stars together with the SRS. The working list of BS was composed at the Pulkovo Observatory and improved by Dr F. P. Scott (the Naval Observatory in Washington). For the whole sky it consists of 5115 stars. Several observatories (Strasbourg, Washington, Kiev, Tashkent, Kharkov, Nikolayev, Santiago, Tokyo and perhaps some others) undertook the meridian observations of these stars. In the U.S.S.R. these observations were made more intensively at the Kiev University Observatory under the guidance of $\mathrm{Dr} \mathrm{N}$. A. Chernega. He intends to compile the 
General Catalogue of BS using all the observations. In reply to our request we have already received the consent from several observatories; the Strasbourg, Tashkent and Nikolayev Observatories have sent the result of BS observations in the form of a final catalogue.

It seems expedient to approve the suggestion of the Kiev Observatory to compile the BS General Catalogue and to ask observatories to send the observational results to Kiev. In the future it is important to continue the meridian observations of all the BS stars, especially in the southern hemisphere, as was recommended in Resolutions of the above-mentioned General Assemblies of the IAU.

\section{Double Stars (DS)}

The resolution No. $17 \mathrm{~b}$ adopted at the $\mathrm{Xth}$ Assembly recommends the meridian observations of the double stars unsuitable for photographic observations. The preliminary working list was compiled at the Pulkovo Observatory; it was improved and supplemented by Scott (1960). The list contains 2292 double stars for the whole sky. For each DS only the brighter star or the preceding one in R.A. is to be observed.

The observations according to this program were made apparently only in Strasbourg (the zones from $+25^{\circ}$ to $+71^{\circ}$ in declination) and in Santiago (the zones from $-47^{\circ}$ to $-90^{\circ}$ ). Prof. P. Lacroute has kindly sent to Pulkovo the results of these observations.

Most of the stars from the DS program were not included in the AGK3 and other photographic catalogues. Study of their proper motions is important for Stellar Astronomy, but in the last 50 years their coordinates have apparently not been determined anywhere, except at the two above mentioned observatories. Thus the above recommendation remains entirely valid.

\section{Latitude Stars (LS)}

This program aims at the reduction of latitude observations, made at various observatories, to a uniform system, which would permit studying in detail a periodic and, later on, a secular polar motion and also non-polar latitude variations of observatories. The LS program has been compiled at the Sternberg Astronomical Institute (Fedorov et al., 1960) for the northern hemisphere. It contains about 3900 stars, 1300 of which are included in the programs of 11 PZTs, and require observations in both R.A. and declination. The remaining 2600 stars are included in the zenith telescope programs of about 20 observatories, and for these stars only declinations need be observed. The importance of meridian observations of the latitude stars is underlined in Resolutions of the X, XI, XII, XIII and XIV IAU Assemblies.

At present the declinations of LS have been determined at the Golosseyevo and Beograd observatories and are being determined at Pulkovo and in Moscow. At the latter two observatories the meridian determination of R.A. of about $1200 \mathrm{PZT}$ stars have been also made. These observations are, of course, not enough for com- 
piling the General Catalogue, therefore the need for participation of other observatories remains still valid.

I welcome the initiative of Japanese astronomers in organizing meridian observations of about 1700 PZT stars according to the list of Yasuda (1971), and wish success to this undertaking.

\section{Zodiacal Stars (ZS)}

The resolution (item 4) of Commission 8 of the XIV Assembly at Brighton (1970) recommends meridian observations of the Zodiacal stars according to the program compiled at the Nikolajev Observatory. This program contains 9518 stars, mainly brighter than $8^{m} .0$, in the zone of $\pm 15^{\circ}$ from the Ecliptic.

The last General Catalogue of about 3500 zodiacal stars was published in Washington in 1940 (Robertson, 1940). Since then only a few meridian observations of these stars have apparently been made. In this connection the suggestion of the Nikolajev Observatory to make a new series of meridian observations of ZS seems to be quite timely, especially in view of modern cosmic researches.

\section{Reference Stars in the Areas with Galaxies of the Pulkovo Program (PS)}

The Pulkovo program of photographing the areas with galaxies (the Deutsch program) was suggested at the U.S.S.R. Astrometrical conference in November 1938 (Zverev, 1939, 1941). The working list of 271 areas for both hemispheres was published by Neuymin (1940). After looking through the numerous test photographs this list for the northern sky (as far as $-5^{\circ}$ declination) was revised and extended at the Pulkovo Observatory (Deutsch et al., 1955). The analogous work for the southern hemisphere was made at the Tashkent Observatory as far as $-25^{\circ}$ declination (Latypov and Fatchikhin, 1955) and more southern declinations at the National Observatory of Chile (Gutierrez Alonso, 1961). Systematic observations according to this program have been made at the U.S.S.R. observatories since 1939. At present photographs of the first epochs have been taken at 12 observatories in both hemispheres with the long-focus and wide-angle astrographs.

The number of reference AGK3R and SRS stars (one star per square degree on the average) is not sufficient for photographs with long-focus instruments (field $\leqslant 2^{\circ} \times 2^{\circ}$ ). Therefore a list of additional reference stars in these areas for the northern sky was compiled at the Sternberg Institute (Moscow) in 1955 (Bugoslavskaya et al., 1955). This list was extended to $-25^{\circ}$ declination at the Tashkent Observatory (Fatchikhin, 1963 ) and recently extended to the south pole at the Pulkovo Observatory (Baturina, 1974).

The whole list of additional reference stars in 301 areas of the Pulkovo program contains 735 stars. Altogether 1630 reference stars (including the AGK3R and SRS) are in these areas, i.e. 5-6 stars per square $2^{\circ} \times 2^{\circ}$ on the average. These stars are to be observed with meridian instruments at the observatories in the northern and southern hemispheres. 
It is desirable that the 735 additional reference stars should be included into the IRS $=$ AGK $3 R+$ SRS program.

\section{References}

Baturina, G. D.: 1974, Trudy 19th Astrometr. Konf., in press.

Bugoslavskaya, E. J., Karimova, D. K., Podobed, V. V., and Jakhontov, K. N.: 1955, Trudy 11th Astrometr. Konf., p. 42.

Deutsch, A. N., Lavdovsky, V. V., and Fatchikhin, N. V.: 1955, Izv. Glav. Astron. Obs. Pulkovo, No. 154, 14.

Fatchikhin, N. V.: 1963, Trudy 15th Astrometr. Konf., p. 161.

Fedorov, E. P., Prodan, Y. J., and Ponomarev, D. N.: 1960, Trudy 14th Astrometr. Konf., p. 139.

Gutierrez Alonso, A.: 1961, Publ. Obs. Nacional Chile, No. 4.

Latypov, A. A. and Fatchikhin, N. V.: 1955, Circ. Tashkent, No. 302.

Neuymin, G. N.: 1940, Kazan University Uchenye Zapiski 100, 86.

Robertson, J.: 1940, Astron. Papers A.E. X, Part 2.

Scott, F. P.: 1967, Trans. IAU XIII A, 73.

Yasuda, H.: 1971, Ann. Tokyo Obs. 8.

Zverev, M. S.: 1939, Astron. Zh. 16, 104.

Zverev, M. S.: 1941, Astron. Zh. 17, 54. 